\title{
A Polynomial Algorithm of Optimum Cutting a Rectangle into Rectangles with Two Heights
}

\author{
M. Z. Arslanov \\ Institute of Problems of Informatics and Control, Almaty, Kazakhstan \\ Email: $\underline{\text { mzarslanov@hotmail.com }}$
}

Received November 1, 2013; revised December 1, 2013; accepted December 8, 2013

Copyright (C) 2014 M. Z. Arslanov. This is an open access article distributed under the Creative Commons Attribution License, which permits unrestricted use, distribution, and reproduction in any medium, provided the original work is properly cited. In accordance of the Creative Commons Attribution License all Copyrights (C) 2014 are reserved for SCIRP and the owner of the intellectual property M. Z. Arslanov. All Copyright (c) 2014 are guarded by law and by SCIRP as a guardian.

\section{ABSTRACT}

We consider the problem of guillotine cutting a rectangular sheet into rectangular pieces with two heights. A polynomial time algorithm for this problem is constructed.

\section{KEYWORDS}

\section{Cutting; Convex Hull; Polynomial Algorithms}

\section{Introduction}

The problems of rectangular packing and cutting are difficult problems of discrete optimization (for references see [1]). For example, the computational complexity of the problem of packing in a rectangular sheet $(A, B)$ the maximum number of equal small rectangles $(c, d)$ (pallet loading problem (PLP)) is unknown. Fast polynomial algorithms $[2,3]$ are known for the problem of optimally guillotine cutting a rectangle $(A, B)$ into rectangles $(c, d)$ where $90^{\circ}$ rotations are allowed. In [4,5] the problem of guillotine cutting a rectangular sheet $(A, B)$ into rectangles $(a, b),(c, d)$ with a minimal trim loss is considered and polynomial algorithms for its solution are presented for the case when the number of occurrences of small rectangles in a cutting pattern is not restricted and rectangles cannot be rotated. Adding a constraint on the number of occurrences of rectangles $(a, b),(c, d)$ complicates the problem. It is not known whether the problem of guillotine cutting a rectangular sheet $(A, B)$ into $m$ rectangles $(a, b)$ and $n$ rectangles $(c, d)$ belongs to $\mathcal{P}$ (the class of the problems which are solvable in polynomial time). The general problem of packing is strongly NP-hard, and in a guillotine case it has pseudopolynomial algorithm of dynamic programming. For integer variant of a problem, from polynomial algorithm of Lenstra [6] for a problem of integer linear programming, it follows a polynomial algorithm for optimum packing of rectangles $\left(a_{i}, b\right), i \in I$ in a rectangle $(A, B)$ with the fixed set $I$. When there are constraints on number of rectangles, the existence of polynomial algorithm is problematic. The matter is that the problem about optimum packing $n_{1}$ rectangles $\left(a_{1}, 1\right), n_{2}$ of rectangles $\left(a_{2}, 1\right), n_{3}$ of rectangles $\left(a_{3}, 1\right)$ into a rectangle $(A, B)$ is equivalent to a problem MSP3 (the problem of packing bins with three lengths), for which the existence of polynomial algorithm is an unsolved problem of the schedulling theory so far [7]. We consider the problem of optimum (i.e with the minimum trim lost) guillotine cutting a rectangle $(A, B)$ into rectangles with two heights $\left(a_{i}, b_{1}\right), i \in I_{1}=1,2, \cdots, n_{1}, \quad\left(a_{i}, b_{2}\right), i \in I_{2}=n_{1}+1, n_{1}+2, \cdots, n_{1}+n_{2}$ without restrictions on the number of copies of each rectangle. Let's designate this problem

$$
\left\{(A, B) ;\left(a_{1}, b_{1}\right), \cdots,\left(a_{n_{1}}, b_{1}\right),\left(a_{n_{1}+1}, b_{2}\right), \cdots,\left(a_{n_{1}+n_{2}}, b_{2}\right)\right\} .
$$

This paper is organized as follows: In Section 2, we consider the properties of a knapsack polygon (the convex hull of the set of feasible solutions of a 2-dimensional knapsack problem). Section 2 is auxiliary for Section 
3 but it is self-interesting for the theory of knapsack problems. In Section 3, we prove the main results about the existence of polynomial algorithm for considered cutting problem.

\section{Some Properties of a Knapsack Polygon}

The set of feasible solutions of a knapsack problem plays an important role for problems of guillotine cutting. In a 2-dimensional case, this set is presented as:

$$
S_{A}=\left\{(x, y) \in Z_{+}^{2} \mid a x+b y \leq A\right\},
$$

where $a, b, A \in Z_{+}=\{0,1,2, \cdots\}$. The convex hull of this set refers to as a knapsack polygon denoted by

$$
P_{A}=c o\left\{(x, y) \in Z_{+}^{2} \mid a x+b y \leq A\right\},
$$

where co denote the convex hull operation.

Let's show, that a knapsack polygon $P_{A}$ can be presented as

$$
P_{A}=\bigoplus_{i=1}^{m} T\left(x_{i}, y_{i}\right)
$$

where $T\left(x_{i}, y_{i}\right)$ is a right triangle with vertices $(0,0),\left(0, y_{i}\right),\left(x_{i}, 0\right), m+1$ is a number of nonzero vertices of $P_{A}$, the symbol $\oplus$ denotes an algebraic sum (the Minkowski sum) of sets:

$$
S_{1} \oplus S_{2}=\left\{z \mid z=x+y, x \in S_{1}, y \in S_{2}\right\} .
$$

If $\left\lfloor\frac{A}{a}\right\rfloor=0$, then it is easy to see, that

$$
P_{A}=T\left(0,\left\lfloor\frac{A}{b}\right\rfloor\right)
$$

is a singular triangle —an interval, connecting points $(0,0),\left(0,\left\lfloor\frac{A}{b}\right\rfloor\right)$. Similarly, if $\left\lfloor\frac{A}{b}\right\rfloor=0$, then

$$
P_{A}=T\left(\left\lfloor\frac{A}{a}\right\rfloor, 0\right) \text {. }
$$

Let's consider the case $\left\lfloor\frac{A}{a}\right\rfloor>0,\left\lfloor\frac{A}{b}\right\rfloor>0$. Let nonzero vertices of $P_{A}$ are sorted by increasing $x$-coordinates.

Then the first vertex is $\left(X_{0}, Y_{0}\right)=\left(0,\left\lfloor\frac{A}{b}\right\rfloor\right)$. Let $\left(X_{1}, Y_{1}\right)$ be the second nonzero vertex of $P_{A}$. Then

$$
P_{A}=T\left(X_{1},\left\lfloor\frac{A}{b}\right\rfloor-Y_{1}\right) \oplus P_{A}^{\prime},
$$

where $P_{A}^{\prime}$ is defined as:

$$
P_{A}^{\prime}=\operatorname{co}\left\{(x, y) \in Z_{+}^{2} \mid a x+b y \leq A-a X_{1}\right\},
$$

and all nonzero vertices of $P_{A}^{\prime}$ are translation of vertices of $P_{A}$, excepting the first, by $\left(-X_{1}, 0\right)$. Applying an induction, we obtain required representation.

Remark. This representation follows from a well-known representation of a convex polygon up to translation by the set of its sides sorted in counter-clockwise or clockwise order [8]. Note also that the Minkowski sum of two polygons can be presented by the union of their sides up to translation.

It is easy to establish the following recurrent equations, connecting coordinates of vertices and parameters of right triangles in the above representation: 


$$
\begin{aligned}
& \left(X_{0}, Y_{0}\right)=(0,\lfloor A b\rfloor) \\
& \left(X_{i}, Y_{i}\right)=\left(\sum_{j=1}^{i} X_{j},\lfloor A b\rfloor-\sum_{j=1}^{i} y_{j}\right) \\
& \left(X_{m}, Y_{m}\right)=(\lfloor A a\rfloor, 0) .
\end{aligned}
$$

Note the following properties of this representation:

$$
\sum_{i=1}^{m} x_{i}=\lfloor A a\rfloor, \sum_{i=1}^{m} y_{i}=\lfloor A b\rfloor .
$$

If triangles are sorted by increasing $\frac{y_{i}}{x_{i}}$ order, then only one triangle can be singular: the first $T_{1}$, if $a x_{1}<b$, then $y_{1}=0$, and the last $T_{m}$, if $b y_{m}<a$, then $x_{m}=0$. If in (1), where right triangles $T\left(x_{i}, y_{i}\right)$ are sorted by increasing $\frac{y_{i}}{x_{i}}$ order, there are no singular triangles, then it is possible to add singular the first and last triangles $T_{0}(0,0), T_{m+1}(0,0)$. Thus, from the beginning we may assume, that $T_{1}, T_{m}$ are singular triangles $T_{1}\left(x_{1}, 0\right)$, $T_{m}\left(0, y_{m}\right)$.

Among other properties note the following property. If $\operatorname{gcd}\left(x_{i}, y_{i}\right)>1$, then

$$
T_{i}\left(x_{i}, y_{i}\right)=\bigoplus_{j=1}^{\operatorname{gcd}\left(x_{i}, y_{i}\right)} T_{i}\left(\frac{x_{i}}{\operatorname{gcd}\left(x_{i}, y_{i}\right)}, \frac{y_{i}}{\operatorname{gcd}\left(x_{i}, y_{i}\right)}\right) .
$$

The similar possibility of further decomposition appears in the case, when boundary of a knapsack polygon besides vertices contains also other points of an integer lattice $Z_{+}^{2}$.

Consider an example of a knapsack polygon:

$$
P_{A}=c o\left(\left\{(x, y) \mid 49 x+80 y \leq 632, x, y \in Z_{+}\right\}\right) .
$$

It has 6 non zero vertices $(0,7),(1,7),(3,6),(8,3),(11,1),(12,0)$ and can be presented by the Minkowski sum of right triangles:

$$
P_{A}=T_{1}(1,0) \oplus T_{2}(2,1) \oplus T_{3}(5,3) \oplus T_{4}(3,2) \oplus T_{5}(1,1),
$$

where $T_{1}(1,0)$ is a singular triangle.

Remark. If we denote by $T^{I}(\alpha, \beta)$ the set of integer points in $T(\alpha, \beta)$ :

$$
T^{I}(\alpha, \beta)=\left\{(x, y) \in Z_{+}^{2} \mid(x, y) \in T(\alpha, \beta)\right\}
$$

then we have the next formula for the set of feasible solutions in a 2-dimensional knapsack problem:

$$
S_{A}=\bigoplus_{i=1}^{m} T^{I}\left(x_{i}, y_{i}\right)
$$

where $P_{A}=\bigoplus_{i=1}^{m} T\left(x_{i}, y_{i}\right)$.

A knapsack polygon is characterized by a triplet of integer non-negative numbers $(a, b, A)$.

Definition 1 A triplet $(a, b, A)$, describing a knapsack polygon, is equivalent to a triplet $\left(a^{\prime}, b^{\prime}, A^{\prime}\right)$ $\left((a, b, A) \sim\left(a^{\prime}, b^{\prime}, A^{\prime}\right)\right)$ if the sets of feasible solutions of corresponding knapsack problems are equal:

$$
a x+b y \leq A \Leftrightarrow a^{\prime} x+b^{\prime} y \leq A^{\prime}, x, y \in Z_{+} .
$$

We say that a triplet $(a, b, A)$ can be decreased, if there exists an equivalent triplet $\left(a^{\prime}, b^{\prime}, A^{\prime}\right) \sim(a, b, A)$, $a^{\prime} \leq a, \quad b^{\prime} \leq b, \quad A^{\prime} \leq A$, and $\left(a^{\prime}, b^{\prime}, A^{\prime}\right) \neq(a, b, A)$.

Neighboring Farey fractions of $\frac{a}{b}[9]$ : 


$$
\begin{gathered}
\frac{a_{2}}{b_{2}}<\frac{a}{b}<\frac{a_{1}}{b_{1}}, \\
a_{1}+a_{2}=a, b_{1}+b_{2}=b, a b_{2}-b a_{2}=a_{1} b-a b_{1}=1 .
\end{gathered}
$$

play an important role for a possibility of decreasing a triplet $(a, b, A), \operatorname{gcd}(a, b)=1$.

It is easy to show, that if $A$ is replaced by the optimum value of a knapsack problem:

$$
A^{*}=\max \left\{a x+b y \mid a x+b y \leq A, x, y \in Z_{+}\right\}
$$

then $(a, b, A) \sim\left(a, b, A^{*}\right)$. If in addition, $A>A^{*}$, then the triplet $(a, b, A)$ can be decreased. Thus, we can assume that $A=r a+s b, r, s \in Z_{+}, \operatorname{gcd}(a, b)=1$. If $r \geq b$ or $s \geq a$, then the triplet $(a, b, A)$ can not be decreased. Let's show, that the triplet $(a, b, a b+r a+s b), a>0, b>0, \operatorname{gcd}(a, b)=1$ can not be decreased. Suppose on the contrary that there exists a triplet $\left(a^{\prime}, b^{\prime}, A^{\prime}\right), a^{\prime}+b^{\prime}<a+b$ :

$$
\begin{aligned}
& a^{\prime} x+b^{\prime} y \leq A^{\prime} \\
& \Leftrightarrow a x+b y \leq a b+r a+s b, x, y \in Z_{+} .
\end{aligned}
$$

Because points

$$
(r+b, s),(r, a+s) \in S=\left\{(x, y) \in Z_{+}^{2} \mid a x+b y \leq a b+r a+s b\right\},
$$

they also belong to

$$
S^{\prime}=\left\{(x, y) \in Z_{+}^{2} \mid a^{\prime} x+b^{\prime} y \leq A^{\prime}\right\}
$$

From here follows, that

$$
a^{\prime}(r+b)+b^{\prime} s \leq A^{\prime}, a^{\prime} r+b^{\prime}(a+s) \leq A^{\prime} .
$$

Let's consider two cases: a) $\frac{a}{b}<\frac{a^{\prime}}{b^{\prime}}$, b) $\frac{a}{b}>\frac{a^{\prime}}{b^{\prime}}$.

a). Let $\frac{a}{b}<\frac{a^{\prime}}{b^{\prime}}$. By virtue of properties of Farey series

$$
\frac{a}{b}<\frac{a_{1}}{b_{1}} \leq \frac{a^{\prime}}{b^{\prime}}, a_{1} b-a b_{1}=1, b^{\prime} a_{1}-a^{\prime} b_{1} \leq 0 .
$$

Consider a point $\left(r+b-b_{1}, s+a_{1}\right)$. It satisfies (2), because

$$
a^{\prime}\left(r+b-b_{1}\right)+b^{\prime}\left(s+a_{1}\right)=a^{\prime}(r+b)+b^{\prime} s+\left(b^{\prime} a_{1}-a^{\prime} b_{1}\right) \leq A^{\prime} .
$$

At the same time, this point does not satisfy (3):

$$
a\left(r+b-b_{1}\right)+b\left(s+a_{1}\right)=a b+r a+s b+\left(b a_{1}-a b_{1}\right)=a b+r a+s b+1 .
$$

That is, triplets $(a, b, a b+r a+s b), \quad\left(a^{\prime}, b^{\prime}, A^{\prime}\right)$ are not equivalent.

b). Similarly, if $\frac{a}{b}>\frac{a^{\prime}}{b^{\prime}}$, then by the properties of Farey series

$$
\frac{a^{\prime}}{b^{\prime}} \leq \frac{a_{2}}{b_{2}} \leq \frac{a}{b}, a b_{2}-a_{2} b=1, a^{\prime} b_{2}-a_{2} b^{\prime} \leq 0
$$

Consider a point $\left(r+b_{2}, s+a-a_{2}\right)$. It satisfies (2), because

$$
a^{\prime}\left(r+b_{2}\right)+b^{\prime}\left(s+a-a_{2}\right)=a^{\prime} r+b^{\prime}(a+s)+\left(a^{\prime} b_{2}-a_{2} b^{\prime}\right) \leq A^{\prime} .
$$

On the other hand, it does not satisfy (3): 


$$
a\left(r+b_{2}\right)+b\left(s+a-a_{2}\right)=a b+r a+s b+\left(a b_{2}-a_{2} b\right)=a b+r a+s b+1 .
$$

That is, in this case triplets $(a, b, a b+r a+s b),\left(a^{\prime}, b^{\prime}, A^{\prime}\right)$ are not equivalent too.

Thus, a question about decreasing a triplet $(a, b, r a+s b)$ is interesting only if $r<b, s<a$.

Lemma 1 Let be given a knapsack problem, the set of feasible solutions of which is given by an inequality

$$
a x+b y \leq A=r a+s b, x, y, r, s \in Z_{+}, r<b, s<a .
$$

The triplet $(a, b, A)$ can not be decreased if and only if $r \geq b_{1}, s \geq a_{2}$.

Proof. We prove this lemma by contradiction. Let, for example, $r<b_{1}$. We shall show, that the triplet $(a, b, A)$ can be decreased. Let's show, that the following inequalities

$$
\begin{aligned}
& a x+b y \leq r a+s b \\
& a_{1} x+b_{1} y \leq r a_{1}+s b_{1}
\end{aligned}
$$

are equivalent.

1) Let $(x, y) \in S=\left\{(x, y) \in Z_{+}^{2} \mid a x+b y \leq r a+s b\right\}$. Let's show, that

$$
(x, y) \in S_{1}=\left\{(x, y) \in Z_{+}^{2} \mid a_{1} x+b_{1} y \leq r a_{1}+s b_{1}\right\} .
$$

Inequality (4) is equivalent to $a(x-r) \leq b(s-y)$. Consider three cases: a) $x=r$, b) $x>r$, c) $x<r$.

a). If $x=r$, then $y \leq s$ and $(x, y) \in S_{1}$.

b). If $x>r$, then $\frac{a}{b} \leq \frac{s-y}{x-r}$. Because $s<a$, then $x-r<b$. By the properties of Farey series, in interval $\left(\frac{a}{b}, \frac{a_{1}}{b_{1}}\right)$ there are no numbers with denominator smaller than $b$, therefore $\frac{a_{1}}{b_{1}} \leq \frac{s-y}{x-r}$, i.e., $\quad(x, y) \in S_{1}$.

c). Let $x<r$. Then $\frac{a}{b} \geq \frac{y-s}{r-x}$. Especially, $\frac{a_{1}}{b_{1}} \geq \frac{y-s}{r-x}$. That is $(x, y) \in S_{1}$.

2) Let $(x, y) \in S_{1}$. We shall show, that $(x, y) \in S$. Inequality (5) can be rewritten as $a_{1}(x-r) \leq b_{1}(s-y)$. Consider three cases: a) $x=r$, b) $x>r$, c) $x<r$.

a). If $x=r$, then $y \leq s$. From here follows, that $(x, y) \in S$.

b). Let $x>r$. Then $\frac{a_{1}}{b_{1}} \leq \frac{s-y}{x-r}$. Especially, $\frac{a}{b} \leq \frac{s-y}{x-r}$, i.e., $(x, y) \in S$.

c). Let $x<r$. Then $\frac{a_{1}}{b_{1}} \geq \frac{y-s}{r-x}$ and $r-x<b_{1}$. Because an interval $\left(\frac{a}{b}, \frac{a_{1}}{b_{1}}\right)$ does not contain rational numbers with a denominator smaller than $b_{1}, \frac{a}{b} \geq \frac{y-s}{r-x}$, i.e., $(x, y) \in S$.

The equivalence of Inequalities (4) and (5) shows a possibility of decreasing the parameters of Inequality (4) if $r<b_{1}$.

A possibility of decreasing the parameters of Inequality (4) if $s<a_{2}$ can be shown similarly.

Let $r \geq b_{1}, s \geq a_{2}$. We shall show, that it is impossible to decrease parameters. Let, on the contrary, the triplet $(a, b, r a+s b)$ can be decreased. Then there is a triplet $\left(a^{\prime}, b^{\prime}, A^{\prime}\right), a^{\prime}<a, b^{\prime}<b$ :

$$
\begin{aligned}
& a x+b y \leq r a+s b \\
& \Leftrightarrow a^{\prime} x+b^{\prime} y \leq A^{\prime}, \quad x, y \in Z_{+} .
\end{aligned}
$$

First of all, $r a^{\prime}+s b^{\prime} \leq A^{\prime}$, as $(r, s)$ is a solution of (6), (7). Consider two possible cases: a) $\frac{a}{b}<\frac{a^{\prime}}{b^{\prime}}$, b) $\frac{a}{b}>\frac{a^{\prime}}{b^{\prime}}$.

a). Let $\frac{a}{b}<\frac{a^{\prime}}{b^{\prime}}$. By the property of numbers of Farey series 


$$
\frac{a}{b}<\frac{a_{1}}{b_{1}} \leq \frac{a^{\prime}}{b^{\prime}} .
$$

But the point $\left(r-b_{1}, s+a_{1}\right)$ does not satisfy (6) because

$$
a\left(r-b_{1}\right)+b\left(a_{1}+s\right)=a r+b s+b a_{1}-a b_{1}=a r+b s+1
$$

and satisfies (7) because $b^{\prime} a_{1}-a^{\prime} b_{1} \leq 0$ and

$$
a^{\prime}\left(r-b_{1}\right)+b^{\prime}\left(a_{1}+s\right)=a^{\prime} r+b^{\prime} s+b^{\prime} a_{1}-a^{\prime} b_{1} \leq A^{\prime} .
$$

That is, in this case decreasing of parameters is impossible.

b) Let $\frac{a}{b}>\frac{a^{\prime}}{b^{\prime}}$. By the property of numbers of Farey series

$$
\frac{a^{\prime}}{b^{\prime}} \leq \frac{a_{2}}{b_{2}} \leq \frac{a}{b}
$$

and $a^{\prime} b_{2}-b^{\prime} a_{2} \leq 0, a b_{2}-b a_{2}=1$. Take a point $x=b_{2}+r, y=s-a_{2}$. Then (7) is carried out:

$$
a^{\prime}\left(b_{2}+r\right)+b^{\prime}\left(s-a_{2}\right)=a^{\prime} r+b^{\prime} s+a^{\prime} b_{2}-b^{\prime} a_{2} \text {. }
$$

At the same time, (6) is not carried out because

$$
a\left(b_{2}+r\right)+b\left(s-a_{2}\right)=a r+b s+a b_{2}-b a_{2}=a r+b s+1 .
$$

The lemma is proved.

\section{Main Results}

It appears that considered problem of optimum guillotine cutting can be decomposed into two subproblems. The following theorem is valid.

Theorem 1 Let $B=r b_{1}+s b_{2}$. Then among optimum cuttings of a problem

$$
\left\{(A, B) ;\left(a_{1}, b_{1}\right), \cdots,\left(a_{n_{1}}, b_{1}\right),\left(a_{n_{1}+1}, b_{2}\right), \cdots,\left(a_{n_{1}+n_{2}}, b_{2}\right)\right\}
$$

there exists cutting the side $B$, such that we have the reduction of an initial problem to two subproblems

$$
\begin{array}{r}
\left\{\left(A, r b_{1}\right) ;\left(a_{1}, b_{1}\right), \cdots,\left(a_{n_{1}}, b_{1}\right),\left(a_{n_{1}+1}, b_{2}\right), \cdots,\left(a_{n_{1}+n_{2}}, b_{2}\right)\right\} . \\
\left\{\left(A, s b_{2}\right) ;\left(a_{1}, b_{1}\right), \cdots,\left(a_{n_{1}}, b_{1}\right),\left(a_{n_{1}+1}, b_{2}\right), \cdots,\left(a_{n_{1}+n_{2}}, b_{2}\right)\right\} .
\end{array}
$$

Proof. Let's prove the theorem by contradiction. Let $(A, B)$ be a rectangle of the minimal area, for which the theorem is not correct. If the first cut for optimum cutting divides the side $A=A_{1}+A_{2}$, the theorem will be fair for rectangles $\left(A_{1}, B\right),\left(A_{2}, B\right)$ and for both of them the first cut divides the side $B$. Let the first cut divides the side $B=B_{1}+B_{2}$. Replace $B_{1}=r_{1} b_{1}+s_{1} b_{2}, B_{2}=r_{2} b_{1}+s_{2} b_{2}$. If $\min \left(r_{1}, s_{1}\right)=0$ or $\min \left(r_{2}, s_{2}\right)=0$, the contradiction easily follows from the reasons, that for rectangles $\left(A, B_{1}\right),\left(A, B_{2}\right)$ the theorem is valid. Let, for example, $\min \left(r_{1}, s_{1}\right)=0$. Then $r \geq r_{1}$ or $s \geq s_{1}$. If $r \geq r_{1}$, we have the first cut $\left(A, r_{1} b_{1}\right),\left(A, B-r_{1} b_{1}\right)$ $=\left(A,\left(r-r_{1}\right) b_{1}+s b_{2}\right)$ and according to the theorem the second rectangle is cut into rectangles $\left(A,\left(r-r_{1}\right) b_{1}\right)$, $\left(A, s b_{2}\right)$. By glueing together rectangles $\left(A, r_{1} b_{1}\right),\left(A,\left(r-r_{1}\right) b_{1}\right)$ we can receive optimum cut $\left(A, r b_{1}\right)$, $\left(A, s b_{2}\right)$. Thus, the desired contradiction can be received only if $\min \left(r_{1}, s_{1}\right)=\min \left(r_{2}, s_{2}\right)=0$. If $r_{1}=0, s_{1}>0, r_{2}>0, s_{2}=0$, or $r_{1}>0, s_{1}=0, r_{2}=0, s_{2}>0$, the contradiction again can be received easily. Really, let we have the first cut $\left(A, r_{2} b_{1}\right),\left(A, s_{1} b_{2}\right)$. Because $r_{2} b_{1}+s_{1} b_{2} \leq r b_{1}+s b_{2}$, then $r_{2} \leq r$ or $s_{1} \leq s$. Let the optimum cut is $\left(A, B-s_{1} b_{2}=r b_{1}+\left(s-s_{1}\right) b_{2}\right),\left(A, s_{1} b_{2}\right)$. Then according to the theorem we have the cut $\left(A, r b_{1}\right),\left(A,\left(s-s_{1}\right) b_{2}\right) \quad\left(A, s_{1} b_{2}\right)$. By sticking together the second and third rectangles we have the optimum cut $\left(A, r b_{1}\right),\left(A, s b_{2}\right)$. It is necessary to consider the case $\min \left(r_{1}+r_{2}, s_{1}+s_{2}\right)=0$. Let, for example, $r_{1}+r_{2}=0$. That is, the optimum cutting has a form $\left(A, s_{1} b_{2}\right),\left(A, s_{2} b_{2}\right)$. If $B=M b_{1} b_{2}+r b_{1}+s b_{2}$ and $M>0$, 
then $B=r b_{1}+\left(M b_{1}+s\right) b_{2}$ and $\min \left(s_{1}, s_{2}\right)<M b_{1}+s$, as $r b_{1}<M b_{1} b_{2}+s b_{2}$ and it is easy to receive the contradiction. Let, for example, $s_{1}<M b_{1}$. For a rectangle $\left(A, r b_{1}+\left(M b_{1}+s-s_{1}\right) b_{2}\right)$ the theorem is valid, therefore we have cutting into rectangles $\left(A, r b_{1}\right),\left(A,\left(M b_{1}+s-s_{1}\right) b_{2}\right),\left(A, s_{1} b_{2}\right)$. It is possible to glue this cutting together with the cutting $\left(A, r b_{1}\right),\left(A,\left(M b_{1}+s\right) b_{2}\right)$. That is, the contradiction results to $M=0, B=r b_{1}+s b_{2}, r<b_{2}, s<b_{1}$. If it is possible to reduce the triplet $\left(b_{1}, b_{2}, r b_{1}+s b_{2}\right)$ to the triplet $\left(b_{1}^{\prime}, b_{2}^{\prime}, r b_{1}^{\prime}+s b_{2}^{\prime}\right)$, by means of the theorem of equivalence [3] then the cutting for a rectangle $\left(A, r b_{1}^{\prime}+s b_{2}^{\prime}\right)$ is decomposed, but then the cutting for a rectangle $\left(A, r b_{1}+s b_{2}\right)$ is decomposed into rectangles $\left(A, r b_{1}\right)$, $\left(A, s b_{2}\right)$ too. Therefore, the triplet $\left(b_{1}, b_{2}, r b_{1}+s b\right)$ cannot be reduced and from the lemma 1 we have $r \geq b_{2}^{\prime}, s \geq b_{1}^{\prime \prime}$, where

$$
\frac{b_{1}^{\prime \prime}}{b_{2}^{\prime \prime}}<\frac{b_{1}}{b_{2}}<\frac{b_{1}^{\prime}}{b_{2}^{\prime}}
$$

are three consecutive members of the Farey series $F_{b 2}$. That is, we have optimum cutting into rectangles $\left(A, s_{1} b_{2}\right), \quad\left(A, s_{2} b_{2}\right)$. But cutting into rectangles $\left(A, r b_{1}\right),\left(A, s b_{2}\right)$ will not be optimum. Because $r b_{1}+s b_{2} \geq s_{1} b_{2}+s_{2} b_{2}$ and the equality is not valid as $\operatorname{gcd}\left(b_{1}, b_{2}\right)=1$, that is $r b_{1}+s b_{2}-1 \geq s_{1} b_{2}+s_{2} b_{2}$. Let's consider rectangle $\left(A, r b_{1}+s b_{2}-1\right)$. If $b_{1}^{\prime}>b_{1}^{\prime \prime}$ (and therefore $b_{2}^{\prime}>b_{2}^{\prime \prime}$ ), we shall receive cutting into $\left(A,\left(r-b_{2}^{\prime \prime}\right) b_{1}\right),\left(A,\left(s+b_{1}^{\prime \prime}\right) b_{2}\right)$ by presenting $r b_{1}+s b_{2}-1$ as $\left(r-b_{2}^{\prime \prime}\right) b_{1}+\left(s+b_{1}^{\prime \prime}\right) b_{2}, r-b_{2}^{\prime \prime}>0$. Because $b_{1} b_{2}^{\prime \prime}=b_{1}^{\prime \prime} b_{2}+1$, we have cutting $\left(A,\left(r-b_{2}^{\prime \prime}\right) b_{1}\right),\left(A, b_{2}^{\prime \prime} b_{1}\right),\left(A, s b_{2}\right)$. Then by gluing together first two rectangles we have required optimum cutting $\left(A, r b_{1}\right),\left(A, s b_{2}\right)$. The case $b_{1}^{\prime}<b_{1}^{\prime \prime}$ is considered similarly. The theorem is proved.

Now the polynomial algorithm for a considered rectangular cutting problem follows from the following reasons. Let's consider the set of feasible solutions for the side $B$

$$
\left\{(x, y) \mid x b_{1}+y b_{2} \leq B, x, y \in Z_{+}=\{0,1,2, \cdots\}\right\}
$$

The convex hull of this set can be presented as the algebraic sum (Minkowski sum) of elementary triangles

$$
\bigoplus_{k=1}^{m} T_{k}\left(X_{k}, Y_{k}\right) \text {. }
$$

Application of the proved theorem and a the theorem 1 of equivalence [3] reduces the initial problem of optimum cutting to the set of problems of optimum cutting the rectangles $\left(A, T_{k}\left(X_{k}, Y_{k}\right)\right)$.

The next lemma follows from area estimations.

Lemma 2 Optimum cutting of a rectangle $\left(A, T_{k}\left(X_{k}, Y_{k}\right)\right)$ can be reduced to the decision of the following knapsack problem

$$
\max \left\{z=\sum_{i \in I_{1}} X_{k} a_{i} x_{i}+\sum_{i \in I_{2}} Y_{k} a_{i} x_{i} \mid \sum_{i \in I_{1} \cup I_{2}} a_{i} x_{i} \leq A\right\} .
$$

Proof. The proof is standard and can be omitted.

Knapsack problem (8) in the case of fixed sets $I_{1}, I_{2}$ can be solved by the well known polynomial algorithm of Lenstra [6] for integer linear programming. Therefore, next lemma is valid.

Lemma 3 For a problem of cutting $\left(A, T_{k}\left(X_{k}, Y_{k}\right)\right)$ into rectangles $\left(a_{i}, b_{1}\right), i \in I_{1}=1,2, \cdots, n_{1}$, $\left(a_{i}, b_{2}\right), i \in I_{2}=n_{1}+1, n_{1}+2, \cdots, n_{1}+n_{2}$, with the fixed sets of indexes $I_{1}, I_{2}$ there exists a polynomial algorithm of optimum cutting.

Proof. If the first cut divide the side $A$, i.e. we have cutting $\left(A_{1}, T_{k}\left(X_{k}, Y_{k}\right)\right),\left(A_{2}, T_{k}\left(X_{k}, Y_{k}\right)\right)$, $A=A_{1}+A_{2}$, then by applying an induction one would receive, that for $\left(A_{1}, T_{k}\left(X_{k}, Y_{k}\right)\right),\left(A_{2}, T_{k}\left(X_{k}, Y_{k}\right)\right)$, the lemma is valid and consequently the validity of the lemma for $\left(A, T_{k}\left(X_{k}, Y_{k}\right)\right)$. Let the first cut divides $T_{k}\left(X_{k}, Y_{k}\right)$. Then we have cutting into two rectangles $\left(A, t_{1}\right),\left(A, t_{2}\right)$. But then by presenting the side $t_{1}$ as the Minkowski sum of elementary triangles we can transform this cutting into cutting with the first cut dividing the side $A$. The lemma is proved.

Uniting all results we obtain the following theorem.

Theorem 2 For a problem of optimum cutting $(A, B)$ into rectangles $\left(a_{i}, b_{1}\right), i \in I_{1}=1,2, \cdots, n_{1}$, 
$\left(a_{i}, b_{2}\right), i \in I_{2}=n_{1}+1, n_{1}+, \cdots, n_{1}+n_{2}$, with the fixed sets of indexes $I_{1}, I_{2}$ there exists a polynomial algorithm.

\section{Conclusion}

As it is said in introduction, the problems of guillotine rectangular cutting belong to the set of problems with pseudopolynomial algorithms. Intuition makes the hypothesis of existence of polynomial algorithm for the problem of optimum guillotine cutting into fixed number of small rectangles probable. In this paper, this hypothesis is justified for the problem of guillotine cutting a rectangular sheet into rectangular pieces with two heights.

\section{REFERENCES}

[1] H. Dyckhoff, “A Typology of Cutting and Packing Problems,” European Journal of Operational Research, Vol. 44, No. 2, 1990, pp. 145-159. http://dx.doi.org/10.1016/0377-2217(90)90350-K

[2] A. G. Tarnowski, J. Terno and G. Scheithauer, “A Polynomial Time Algorithm for the Guillotine Pallet Loading Problem,” INFOR, Vol. 32, 1994, pp. 275-287.

[3] M. Z. Arslanov, “A Polynomial Algorithm for One Problem of Guillotine Cutting,” Operations Research Letters, Vol. 35, No. 5, 2007, pp. 636-644. http://dx.doi.org/10.1016/j.orl.2006.12.003

[4] A. G. Tarnowski, “Advanced Polynomial Time Algorithm for Guillotine Generalized Pallet Loading Problem,” In: The International Scientific Collection: Decision Making under Conditions of Uncertainty (Cutting-Packing Problems), Ufa State Aviation Technical University, 1997, pp. 93-124.

[5] E. Girlich and A. G. Tarnowski, “On Polynomial Solvability of Two Multiprocessor Scheduling Problems,” Mathematical Methods of Operations Research, Vol. 50, No. 1, 1999, pp. 27-51.

[6] H. W. Lenstra Jr., “Integer Programming with a Fixed Number of Variables,” Mathematics of Operations Research, Vol. 8, No. 4, 1983, pp. 538-548.

[7] S. T. McCormick, S. R. Smallwood and F. C. R. Spieksma, “A Polynomial Algorithm for Multiprocessor Scheduling with Two Job Lengths,” Mathematics of Operations Research, Vol. 26, No. 1, 2001, pp. 31-49.

[8] L. A. Lyusternik, “Convex Figures and Polyhedra,” Dover Publications, New York, 1963.

[9] R. K. Guy, “The Book of Numbers,” Springer-Verlag, New York, 1996. 